\title{
Novel energy optimization approach for electrical vehicles in a smart city
}

\author{
Flah Aymen ${ }^{1, *}$, chokri mahmoudi ${ }^{2}$ and Lassaad Sbita ${ }^{1}$ \\ 1 National school of engineering of Gabès, University of Gabès, Tunisia 1; flahaymening@yahoo.fr \\ 2 Affiliation 2; e-mail@e-mail.com \\ * Correspondence: flahaymening@yahoo.fr; Tel.: +21621104838
}

\begin{abstract}
:
Smart cities and smart technologies have been incorporated into several axes to increase comfort of life. The connected buildings concept was introduced for this reason. However, it was utilized in power management the for a better organizing, greater buildings management, and monetary savings. Cars technologies and number of vehicles are also involved; Nowadays, each house has at least one car. Technological evolution helped to make those cars intelligent and connected. In the latest versions, the majority of those cars were equipped with several sensors, several communication protocols and a principal electrical control unit (ECU), especially for the electric vehicle model. This type or architecture was an essential element in a smart city, thus, it helps to manage power and decide when a vehicle needs to be charged. Based on the smart city concept and using possible network communication between buildings and vehicles, EVs can share their own information related to the power experience on a specific path. This information, can be gathered in a gigantic database and used for managing the power inside these vehicles. In this field, we propose in this paper a new approach for power management inside an electrical vehicle based on bicommunication between vehicles and buildings. The proposed approach is founded on two essential parts; the first is related to vehicles' classification and buildings' recommendation according to different car positions. Two algorithms, related to the SVC and neural network was employed in this work for implementing the final process. Different possibilities and situations were discussed for this approach. The proposed method was tested and validated using Simulink/Matlab application. The state of charge of the used battery was compared at the end of this work, for two specified cases, for showing the contribution of this approach.
\end{abstract}

Keywords: Smart city, energy management, electric vehicle, optimization, classification, state of charge, intelligence.

\section{Introduction}

Electrical power is one of the important source of energy in our daily life. It is used in all areas for rapid development of the economy. The efficiency of the related production systems and power transmission system is very essential for the sustainable development of the economy and environmental protection ${ }^{1}$. Saving energies is also a goal for consumer and energies producer. Many researchers were established in this context. Renewable energy was introduced in this field in order to minimize the electrical power production from the combustion based generators. The related 
technologies were rapidly developed in order to extract the maximum of energies from the natural sources in extreme conditions ${ }^{2}$.

Electrical energies were also used in transportation systems like high-way train (HWT) and electrical vehicles (EVs). Meanwhile, the problem is often related to the electric vehicle and its power saving problem. Also, power losses minimization problem inside cars presents a serious challenge for the researchers. Even with these problems and according to ${ }^{3}$, EVs have gained renewed attention in the vehicle market and the global market. EV consumers are expected to reach in 2030 three times the amount recorded in 2011. This is due to the high technologies' performance used for battery and its consequence on vehicle autonomy. Studies were not stopped at EVs development, but aim were to make an EV comfort in relation to the autonomy $(\mathrm{Au})$, and this is a serious challenge. Based on various statistics about different EVs, as it is exposed in ${ }^{4},{ }^{5}$ and ${ }^{6}$, we present in the table (1) the classification for a various EV model in relation to $(\mathrm{Au})$. Even the new statistics compared to a previous version, make users afraid when to decide to use EVs for a long distance. According to various tests seeking equivalence between the electrical and the combustible energetic ingesting inside an EV car, we have concluded that $10 \mathrm{~kW}-\mathrm{H} / 100 \mathrm{Km}$ is approximately equivalent to $1 \mathrm{~L} / 100 \mathrm{Km}$ and this valid on the high way road. So, saving energy will expand the autonomy and then save money.

Table 1. Related autonomy and battery power for various cars type ${ }^{7}$

\begin{tabular}{cccc}
\hline EV Model & Autonomy & Battery & Kw-H/100Km \\
\hline Tesla* $^{*} 542 \mathrm{Km}$ & $85 \mathrm{Kw}$ & 20.9 \\
\hline Chevrolet & $382 \mathrm{Km}$ & $16 \mathrm{Kw}$ & 23.5 \\
\hline Hyundai & $200 \mathrm{Km}$ & $28 \mathrm{Kw}$ & 17.2 \\
\hline Ford Focus & $185 \mathrm{Km}$ & $33.5 \mathrm{~kW}$ & 18.2 \\
\hline \multicolumn{4}{c}{${ }^{*}$ the best Ev performance in 2018 }
\end{tabular}

To increase the vehicle autonomy, managing the power inside car was a solution to save energy, the idea is based on the minimization of the electrical consumed power as much as its permitted when the car is in movement ${ }^{8},{ }^{9}$. Here, authors have exposed a useful solution for managing power, their approach consists to provide the EV by a hybrid charging system, which contains an ICE engine and an electrical generator. In this case, the battery can be charged even the car is in motion. However, the problem is always related to the internal system complexity and the high prices of this car model. Another solution was introduced based on renewable energy sources like solar energy ${ }^{10}$ or the obtained power during deceleration phase called also regenerative braking ${ }^{11}$. Those systems were proficient but the quantity of the obtained power is not sufficient, especially in a high way road. According to ${ }^{12}$, regenerative braking systems can't pass $5 \%$ of the battery needed power when it will be fully charged. New charging systems based on Wireless circuit founded a suitable solution on high way road case. In ${ }^{13}$ authors expose this system and describe its advantages and inconveniences. The problems are also related to car speed and charging system's complexity. The recharge level depends on the car speed and road length. All these technics for charging vehicles were acceptable and efficient, however, the same problem remains always attached to each model and its relation to system complexity.

Related to the autonomy amelioration objective, several software approaches and strategies were proposed in the literature in order to improve the energetic performance of the vehicle. In ${ }^{14}$ authors used intelligent technics for power optimizing in a hybrid vehicle. Also, in ${ }^{15}$, authors' work was based on fuzzy technics for online power managing inside vehicle. An important review was exposed in ${ }^{16}$ where authors developed used control strategies into two categories: the first one is based on fix rules and the second focuses on optimization method. Related to same context, our proposed approach is introduced and its main idea is power optimization.

The added value of this approach is that the searched optimum parameter will be calculated outside the vehicle taking advantage of smart city. The high technologies used inside a smart city will 
be a benefit for our proposed technic. Installed equipment such as sensors, cameras, antennas and the network communication capability were important for security, building management and power control. For example, in Singapore, smart city helped saving 5 to $50 \%$ of the used power and this is according to ${ }^{17}$. Referring to ${ }^{1}$ and 18 ,smart cities can be a benefit to save energy in relation to EVs application. Taking advantage of smart cities and possible network communication between buildings and vehicles, it is possible to save energy inside EV also.

If each vehicle can be identified and shares their parameters related to the weight, speed, state of charge of the battery, driving method, vehicle position on map and some other information, we can gather all of those data and classify these vehicles in specific classes. It is possible then to build the "energetic experience" of each vehicle on a specific road. According to those data, we can calculate and identify the optimum case. In this specific road, the existing building can record data, classify it and calculate optimal parameters. Then, each building can determine optimal driving method according to the closet road condistions as hill climb, bends, traffic jam etc....

Hundreds of vehicles can drive every day in front of those buidings. If we gather all driving information we can calculate the optimal driving solution according to each specific situation. Then each building informs the vehicle with best decisions, which is equivalent to the optimum relation between energy and acceleration. That information can be exchanged between buildings to obtain optimal control method for long distance and, thus, we optimize the energy of the vehicle. Each vehicle can also optimize its own control system. The driver control method is not the same for all the drivers. The total energy consumption is related to driver habits. In relation to the vehicle position on the road, as bends or hill climbs, the driving mode will have a direct impact on energetic efficiency and on the vehicle autonomy. Essentially, two algorithms will be used in this work, first one will be related to the vehicles classification and second will define optimum solution according to the existing database.

The proposed approach was tested on a simulation work and the obtained results showing an energetic gain with this novel methodology.

The paper is therefore organized as follow. After this introduction section, the electrical vehicle model is exposed defining interior components and the objective energetic expressions. Next, the problem is formulated and the proposed solution is detailed and explained. In this section, the relation of the smart city concept and the vehicle power management is summarized. Before the conclusion and in the light of this circumstance and for proven the successes of the proposed approach we have presented simulation results for a simple example of six vehicles placed in a smart city.

\section{Electrical vehicle and the basic components}

Electrical vehicles were classified into two categories, the hybrid, and the pure electric vehicle. Each model was characterized by its advantages and problems. Refers to ${ }^{19}$ the pure electrical vehicles are more friendly to the environment. In this way, the world tendency is using these EV categories. Therefore, researches were not stopped here, and the new EV versions are aiming to optimizing battery technologies, charge technics, than main motors and to the internal control algorithms. The objective is maximizing injected power from the external sources and for minimizing energy losses inside car. So, we will focus in this paper on pure EV model. Figure (1), exposes this EV and its components. The basic version is composed of batteries system which will be connected to inverter to feed the motor. A principle controller organizes this overall system. 


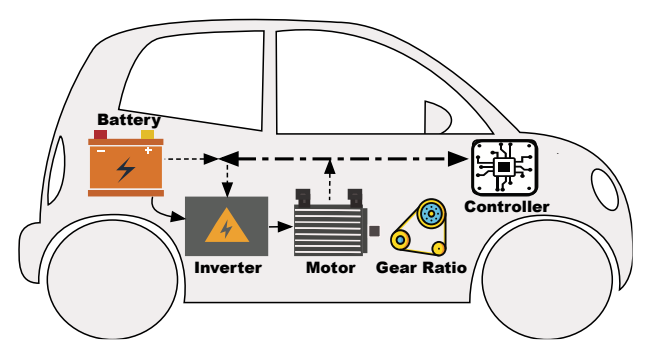

Figure 1. EV and its basic components

This controller is the supervisor of the power source inside the car. It will calculate the needed and used power based on real time driver demand. Referring to various researches, the needed and the consumed power can be evaluated or estimated using the analytical models of the car components. Several other sensors were used in the new version of EV and their role is very important for finishing the car control phase.

\subsection{Modeling of principal components for an electric vehicle}

In this work all, the results are obtained after using the mathematical models of the EV components and related to vehicle torque " $\mathrm{C}_{\mathrm{v}}$ ", total Mass " $\mathrm{Mv}_{\mathrm{v}}$ ", electric motor power " $\mathrm{P}_{\mathrm{m}}$ ", battery Power "Pbatt", battery current "Ibatt" and instantaneous battery state of charge "SOC(K)", respectively in equations (1) to (6).

$$
\begin{aligned}
& C_{v}=r_{w}\left[f_{r} M_{v} g \cos ()+M_{v} g \sin ()+\frac{1}{2} C_{d} A_{v} V_{v}^{2}\right], \\
& M_{v} \square m_{v 0}+m_{b a}+m_{e m} \text {, } \\
& P_{m}=\left(\begin{array}{ll}
C_{m} &
\end{array}\right) /{ }_{m}, \\
& P_{\text {batt }}=V_{o c} I_{b a t t} I_{b a t t}^{2} R_{b a t t}, \\
& I_{b a t t}=\frac{V_{o c} \sqrt{V_{o c}^{2} 4 R_{b a t t} P_{b a t t}}}{2 R_{b a t t}} \\
& \operatorname{SOC}(k+1)=\operatorname{SOC}(k)\left[\frac{I_{\text {batt } t}}{Q_{c}}\right]^{\prime}
\end{aligned}
$$

All of the used notation was summarized in Appendix A

\subsection{Related Energy expressions for an electric vehicle}

Refers to ${ }^{20}$, the state of charge of an electric vehicle is related to several parameters and the driver acceleration form will affect battery SOC. Authors proved in this reference, that the acceleration is inversely proportional to the remaining battery SOC. Equation (7) presents the percentage of the state of charge of a lithium battery, fully charged at the departure, in function of an acceleration value, noted by (Acc). We indicate by SOC(0), the initial state of charge of the used battery. $\alpha_{b a t}$ is related to the battery model.

$$
\operatorname{SOC}(k)=\quad{ }_{b a t}(\operatorname{Acc}(k))+\operatorname{SOC}(0),
$$

Also in ${ }^{21}$, authors presented a mathematical equation, which proves that the needed power for driving an electric vehicle compose the vehicle speed factor and then this expression can be presented using the vehicle acceleration factor. Equation (8), presents the power expression to drive the vehicle at speed $\mathrm{V}_{\mathrm{v}}$. We indicate by $\mathrm{F}_{\mathrm{t}}$, the total propulsion force. This variable holds a lot of factors related to the vehicle weight, external forces and other as it is exposed in equation (9). More explanation is given in the joined reference ${ }^{22}$. 


$$
\begin{gathered}
P_{b a t t}(t) \simeq P(t)=F_{t} V_{v}(t), \\
F_{t}=M_{v} g \sin ()+\frac{1}{2} A_{v}\left(V_{v} \quad V_{w}\right)+M_{v} g f_{r} \cos ()+f_{m} M_{v} A c c,
\end{gathered}
$$

It is clear that needed electrical power for driving the vehicle can be expressed also with the vehicle acceleration ratio. In the table (2), we present an example of the propulsion power needed for a car acceleration, weighted $500 \mathrm{Kg}$, from $0 \mathrm{Km} / \mathrm{h}$ to $70 \mathrm{Km} / \mathrm{h}$ in two different acceleration ratios.

Table 2. Propulsion Power of an example of a vehicle of $500 \mathrm{Kg}$ with two different accelerations ratio

\begin{tabular}{lll}
\hline Vehicle Mass & $500 \mathrm{~kg}$ & $500 \mathrm{~kg}$ \\
Acceleration 0 to $70 \mathrm{Km} / \mathrm{h}$ at & $\mathbf{7 ~ m} / \mathrm{s}^{2}$ & $\mathbf{3 , 5} \mathbf{m} / \mathrm{s}^{2}$ \\
Air density & $1,225 \mathrm{~kg} / \mathrm{m}^{3}$ & $1,225 \mathrm{~kg} / \mathrm{m}^{3}$ \\
Wind speed & $0 \mathrm{~m} / \mathrm{s}$ & $0 \mathrm{~m} / \mathrm{s}$ \\
The coefficient of rolling resistance & 0 & 0 \\
Aerodynamic drag coefficient & 0.3 & 0.3 \\
Average needed power & $\mathbf{4 7 , 3} \mathbf{~ k W}$ & $\mathbf{4 3 , 1} \mathbf{k W}$ \\
Initial State of charge & $100 \%$ & $100 \%$ \\
Final State of charge & $\mathbf{9 9 . 9 7 \%}$ & $\mathbf{9 9 . 9 9} \%$ \\
\hline
\end{tabular}

From table (2), we can conclude that more the acceleration ratio is important, more needed power increase and then the state of charge of the battery decrease. So, in a city where a traffic jam is frequent and nervous driving is common, we can decrease rapidly the battery state of charge without an efficient output. Also, for some specific bends, a high acceleration form is not recommended because it is useless due to security bending limits.

\section{Proposed Power management approach for an EV in a relation to the Smart city concept}

The smart city concept has been introduced for several objectives. The principle impartial is related to the energetic optimization. Supervising and controlling the electrical power inside the cities can be a benefit for managing the generation part ${ }^{23}$. Researchers have used the "smart" expression due to the possibility of exchanging information between houses and buildings. This is due to the several sensors and camera used in the cities and due to the internet network connection 24 .

Referring to various researchers as 17,25 and 26 , smart city concept is characterized by numerous advantages, related to the energy saving and the security optimizing, however, the principle disadvantages is attached to the public provision of the own data on the internet network.

Based on smart city specifications, the idea is to use the possible network connections between buildings for helping electrical vehicle saving energy on the road and then increasing their battery autonomy. Sharing specific data from vehicles and from the building will be the key to optimizing the power inside cars.

In figure (1), we expose three specific road signs which indicate that the car must minimize its speed which means that high acceleration form is not recommended. If we suppose that in each signed zone we have a supervision system which takes vehicle speed and all related information, to build a database of information specific for this zone. This database will be then used for any special objective. Our objective here is to find the optimal energetic experience form.

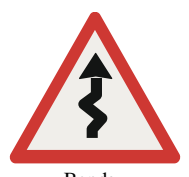

Bends

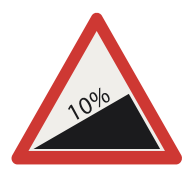

Hill climb

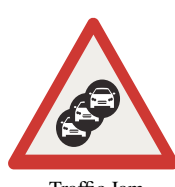

Traffic Jam

Figure 1. Three Road signs which significate that the high acceleration form is useless 


\subsection{Problem formulation}

As it is mentioned in the previous section, the needed electrical power for an electric vehicle is related to the acceleration form and this component will affect the state of charge of used battery. In the given example in table (2), we have exposed two different cases. In first case, driver can reach the needed speed in $10 \mathrm{~s}$ however in second case, the driver can reach the same speed after $20 \mathrm{~s}$. In some areas, the vehicle speed is not useful. As in a traffic jam, the vehicle speed is not important and it can be classified as a minor speed. However, the driver can give several high accelerations forms. This is will cost power loss and this driving form will harm the battery autonomy. Also, in other situations and in a hill climb, the driver can give some high acceleration form without an immediate speed variation. This case will also degrade the battery state of charge. In some additional cases, where we road gets some bends, high speed can be dangerous and a high acceleration form is not accepted. This driving mode will also affect the battery state of charge without an efficient output from the vehicle. Figure (2), exposes an example of six vehicles placed a road which contains the three describer previous zones. In each specific zone, we have located a proportional building.

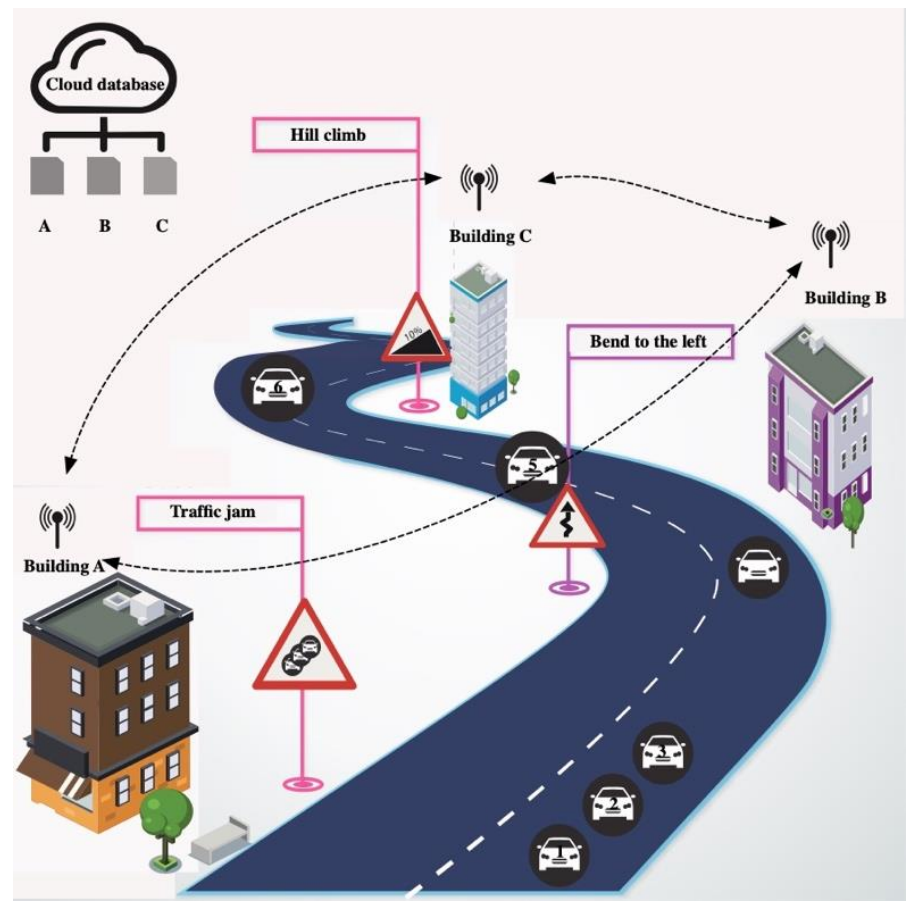

Figure 2. Three different cases for a useless high acceleration form on the road

As it is indicated in the figure (2), the six cars pass through three specific road situations as it is indicated before. Supposing, that sixth, fifth, fourth and third car pass through this trajectory every day and drivers know bends and hill climb positions. The driver's methods will be updated automatically every day. The speed and the acceleration form for each driver will be adjusted according to the known situation on the road and then the energetic consumption will be different according to this driving modification. However, for the second and the first car, the drivers do not know this trajectory and they can give some useless acceleration form.

If the drivers of the first and the second cars, know the driving method of the other cars, they will follow this form, because it is the optimal driving method by the other drivers and it is the economic one.

In the table (3), we give an example of the acceleration and speeds ratio for the common driving methods for a similar case on a road. Those statistics were taken from ${ }^{27}, 28$. 
Table 3: Maximum speeds and acceleration values in a various road situation in a city

\begin{tabular}{ccc} 
& Acceleration ratio & Speed value \\
\hline Traffic jam & $3 \mathrm{~m} / \mathrm{s} 2$ & $15 \mathrm{Km} / \mathrm{H}$ \\
Bends of $30^{\circ}$ & $2 \mathrm{~m} / \mathrm{s} 2$ & $60 \mathrm{~km} / \mathrm{H}$ \\
Bends of $60^{\circ}$ & $2 \mathrm{~m} / \mathrm{s} 2$ & $40 \mathrm{Km} / \mathrm{H}$ \\
Hill climb of $20 \%$ & $1 \mathrm{~m} / \mathrm{s} 2$ & $30 \mathrm{Km} / \mathrm{H}$ \\
Hill climb of $10 \%$ & $2 \mathrm{~m} / \mathrm{s} 2$ & $40 \mathrm{~km} / \mathrm{H}$ \\
\hline
\end{tabular}

So, if a vehicle is driven with a highest speed or acceleration values in a similar condition, more energy will be extracted from source without a perceptible reaction from the vehicle. So, to minimize the quantity of used battery power, we must control the acceleration form as it is exposed previously. The objective function, that will be optimized is related to equation (10).

$$
J^{*}=\min P_{b a t t}(t) d t=\min f(A c c(t), t) d t
$$

\subsection{Explanation of the Proposed solution}

As we have explained previously, we can conclude that the two parameters related to speed and to vehicle acceleration must be controlled or supervised in order to control the quantity of used energy inside vehicle and to guarantee the driver safety. In general, the driving standards law forces the driver to follow the road signs. This will guarantee the driver safety on the road, but those signs will not recommend the driver to use the economic driving method.

According to the latest technologies used inside electric vehicles, such as smart sensors, GPS antennas, smart cameras, the advanced connection technologies as high-speed Bluetooth 5.0, wireless technology, direct Wi-Fi and high-speed calculator, the proposed solution is built.

Effectively, the idea is based on the information shared between vehicles and buildings. If each vehicle shares their own energetic experience and driving method information, the other cars can use those data for adjusting their own parameters. Then, each vehicle can operate with the best driving method, which is equivalent to the optimum energetic consumption form in a specific place.

Referring to figure (2), the cars numbered from six to four share their data, the first and the second cars will use the best performance related to one of four previous cars.

The best car performance will be related to the optimum consumed energy and this will be obtained only after two specific steps. The first one is related to the vehicle classification and the second one will be related to the optimization phase. More explanation for those steps will be uncovered next.

\subsubsection{Vehicle classification}

As we focus on the energetic consumption mode, the proposed method will pass firstly by a classification phase. This step will classify vehicles according to several parameters about the vehicle status. In Table (4), we explain and code those data. We have considered four different parameters, related to speed, acceleration ratios, weight of the vehicle and to the consumed power. As we have present in equation (9), the weight of the vehicle and the external forces can increase the demand of power by the vehicle.

Table 4: Shared data type and proportional class

\begin{tabular}{|c|c|c|c|c|c|c|c|c|c|c|c|c|}
\hline \multicolumn{3}{|c|}{ Data Classification } & C 1 & $\mathrm{C} 2$ & $\mathrm{C} 3$ & $\mathrm{C} 4$ & $\mathrm{C} 5$ & C77 & C78 & C79 & $\mathrm{C} 80$ & C81 \\
\hline \multirow{3}{*}{ Acc $\%$} & Acc1 & {$\left[\begin{array}{ll}0 & 0.3\end{array}\right]$} & & & & & & & & & & \\
\hline & Acc2 & & & & & & & & & & & \\
\hline & Acc3 & {$[0.701[$} & & & & & & & & & & \\
\hline \multirow{2}{*}{$\mathrm{P}(\mathrm{kW})$} & P1 & $<20$ & & & & & & & & & & \\
\hline & P2 & $20<. .<50$ & & & & & & & & & & \\
\hline
\end{tabular}




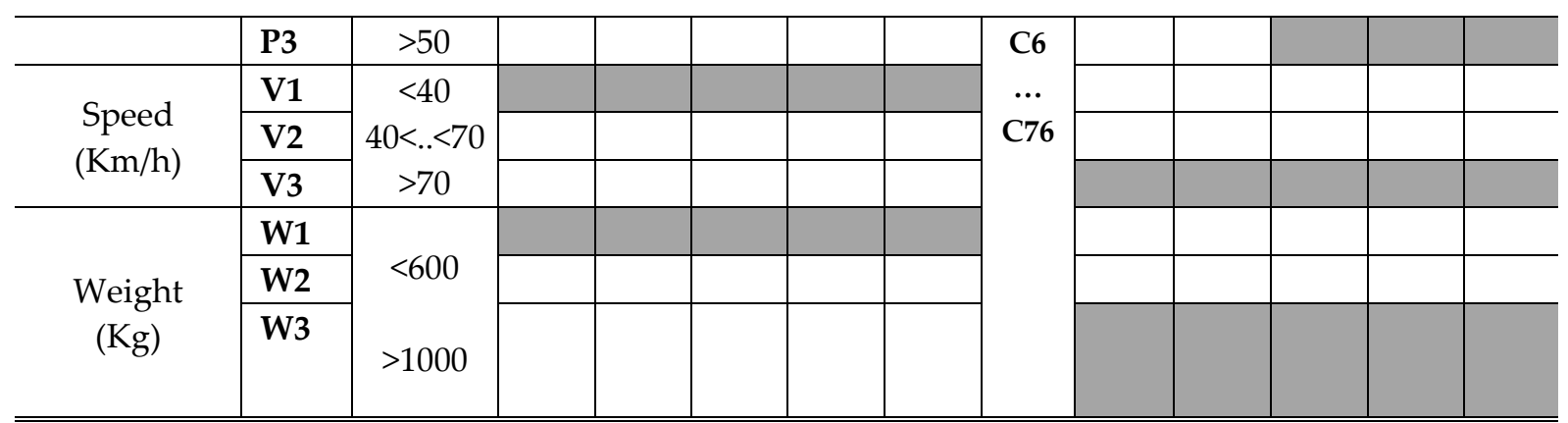

We indicate by $\mathrm{C} 1$ to $\mathrm{C} 81$, the classes which correspond to each possible case. We divided all these parameters into three intervals. For the acceleration form, we coded by "Acc1" the interval of acceleration between $0 \%$ and $30 \%$. We have coded by "P1" the quantity of power less than $20 \mathrm{Kw} / \mathrm{h}$. If the car speed is superior then $70(\mathrm{Km} / \mathrm{h})$ the corresponding code is "V3" and by "V1" if the vehicle speed is under $30(\mathrm{Km} / \mathrm{h})$, else, the speed code is V2. We have coded the vehicle weight by "W1" to "W3" if the vehicle weight increases from $600 \mathrm{Kg}$ to more than $1000 \mathrm{Kg}$.

All of those data will be shared by each car and then it is possible to build a big database which contains the energetic experience of each car.

\subsubsection{Vehicle and building communication: the principle of operation and explanation}

As it is described before, each vehicle will share their own data to a specific destination in order to use that information for adjusting some energetic experiences for other EVs. The principle of the proposed idea is based on the advantages of the smart city, where each building can connect to the other using the internet connection. Refers to figure (2), building A, B and C can connect and exchange information.

For example, if building " $\mathrm{A}$ " detects the vehicle number " 1 " and calculate its speed, building " $B$ " can identify the arrival time of this vehicle. This is possible only if building " $A$ " informs building "B" about this information. Basing on this principle, we can use the building for informing vehicles about the optimal energy form for a specific condition.

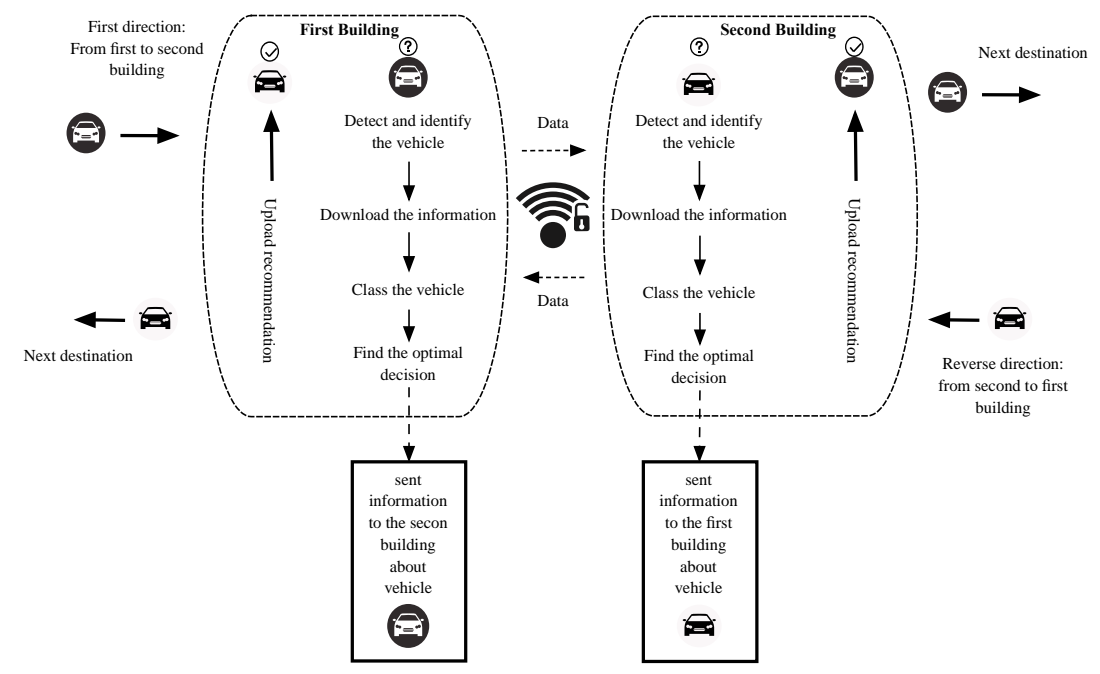

Figure 3. The communication protocol between buildings about changing information related to the vehicles

In figure (3), we explain the communication protocol between two buildings and the possibility to generalize this principle on other houses. However, there is a specific condition that must be verified. This condition is related to the building position and if it is important. Here we are interested only by the building which are close to the bends, hill climbs and where there is a traffic jam as schools, industry, etc... 
We suppose that the white vehicle direction is from the first building "B.1" to the second "B.2". This car is a new one in the database it is unknown by the two structures. For this vehicle trajectory, this car will be detected firstly by "B.1" and then a software application will code and identify it. Then this car will be classified into the corresponding class according to the table (4). Finally, the software application will find the optimal energetic form for this case. The car class or category will be shared with the other buildings, that have information about the future status of the road. Then it is possible to recommend for this vehicle the best driving method in each critical road position.

Referring to figure (2), building " $\mathrm{A}$ " will detect and identify the car number " 1 ". Those results will be sent to the building " $B$ " and " $C$ ". Building " $B$ " will find, from the existing database, the best driving method which is equivalent to the optimal energetic form for driving through the bend. Building " $\mathrm{C}$ " will recommend to this vehicle the best driving method corresponding to the hill climb. We notice that in each phase, the database will be updated according to the novel status of each car.

\subsubsection{Algorithm running principle}

We have transformed this idea into a flowchart for describing the principle of this concept. Figure (4), explains the idea. If the running vehicle is detected by a building " $\mathrm{i}$ ", this structure will ask the vehicle parameters and information.

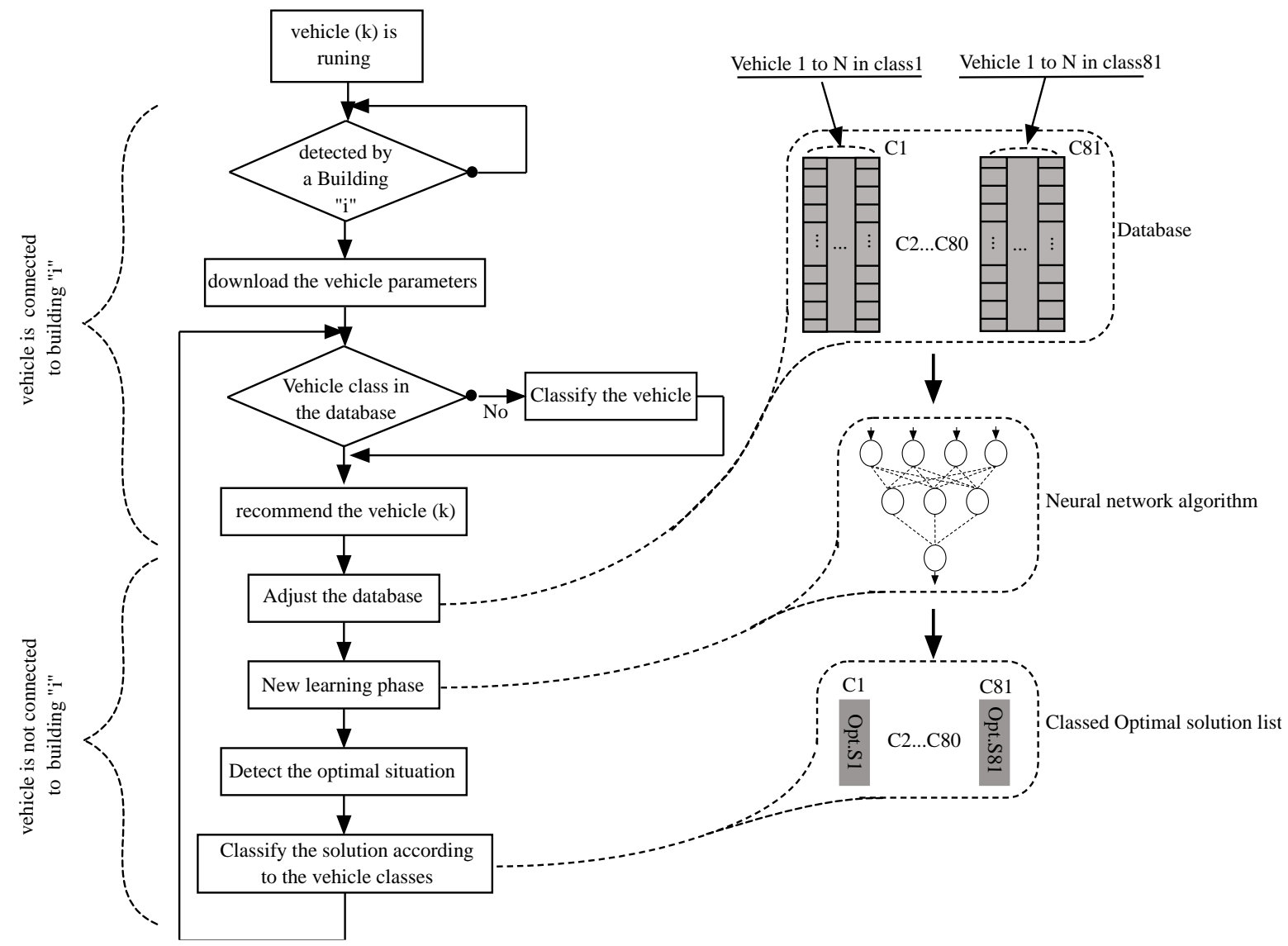

Figure 4. Flowchart of the proposed algorithm

It is possible that this vehicle is in the database list and it is classed before, then it is simple to find the vehicle recommendation according to the vehicle trajectory, "each building can calculate the best solution according to the road situation for each vehicle class". Else, the new vehicle will be added to the database and it will be classified. In the same time, the database will be adjusted automatically and the new optimal solution for each class will be adjusted by a specific algorithm using neural network technics. The new solution will be also classified for each specific case and transferred to the buildings for any possible request. Specific 


\subsubsection{The principle of vehicle information treatment for the learning phase}

The vehicle data will be enormous and the management of this amount of data will be difficult and needs powerful calculators. Therefore, each building will focus on its specific zone information. The principle of using vehicle info will be as it is described in figure (5). As we have used a neural network algorithm for the learning phase, the data that will be used must be related to the specific zone and handled by building "i" and the zone before. After the learning phase, a reference mathematical model "optimal model" will be generated and it is possible to estimate the best acceleration form in the desired zone from the vehicle information before entrance in the specific structure limit.

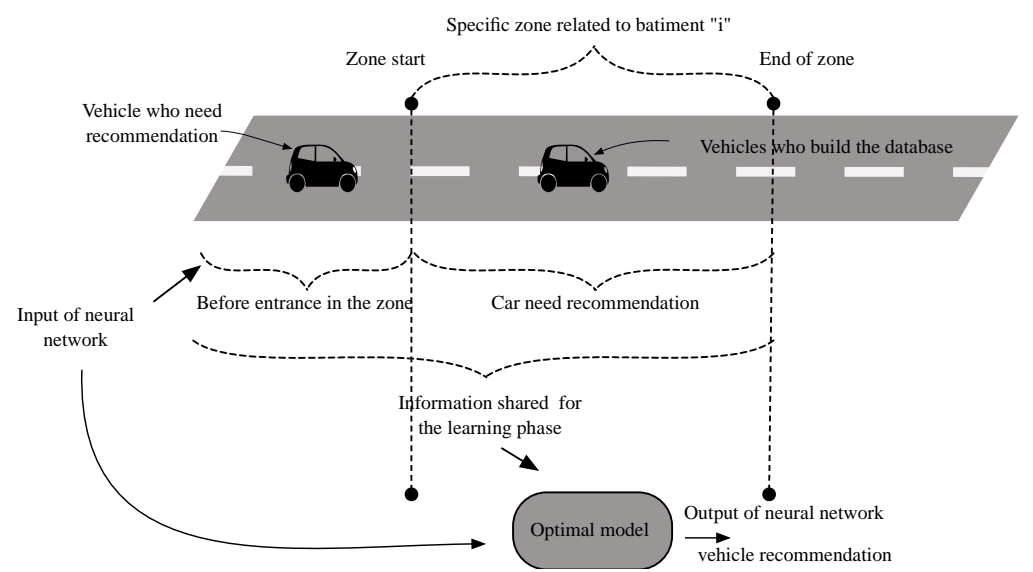

Figure 5. The principle of the learning phase and vehicle zone recommendation

\section{Results}

In this part, we expose the simulation results related to the presented work. To prove the effectiveness of this approach we have taken an example of six vehicles who will across a specific region which contains three specific situations as traffic jam, bends, and a hill climb. In the results, we will expose the difference between the two cases. The first case which corresponding to one vehicle which doesn't use the energetic recommendation. The second case is for the same vehicle which accepts the energetic recommendation. We indicate by the energetic recommendation, the best driving method which is equivalent to the optimal energetic consumption form.

This section will be divided into four subsections. In the first one, we will detail the classification phase results. Next, we will present the user database. The next section exposes the learning phase results and shows the optimal solution for a specific class. In this final section, we detail the energetic gain according to one example of vehicle.

\subsection{Classification Problem}

As it is mentioned in the flowchart, the first and the final step that will be executed by the algorithm related to the vehicle and the results classification. In this example, we used the support vector classification (SVC) theory due to the application rapidity needed. Similar works prove the SVC rapidity for a comparable application $29,30$.

For this demonstration, we have used four cars as it is mentioned in the table (5). In the same table, we have presented the classification results related to each vehicle in each specific position. The classification scores were related to the first classification problem in the proposed flowchart.

Table 5: Used cars parameters and related classification results

\begin{tabular}{lccccc} 
& & Vehicle 3 & Vehicle 4 & Vehicle 5 & Vehicle 6 \\
\hline $\begin{array}{l}\text { Used data for } \\
\text { classification }\end{array}$ & $\begin{array}{c}\text { Weight (kg) } \\
\text { speed } \\
\text { Power }\end{array}$ & 600 & 550 & 580 & 600 \\
& & & In figure (6) & \\
\hline
\end{tabular}




\begin{tabular}{cccccc}
\hline & Acceleration & \multicolumn{4}{c}{ In Figure (6) } \\
\hline \hline SVC-class & B1 & $\mathrm{C} 1$ & $\mathrm{C} 1$ & $\mathrm{C} 1$ & $\mathrm{C} 1$ \\
according to & B2 & $\mathrm{C} 1$ & $\mathrm{C} 4$ & $\mathrm{C} 1$ & $\mathrm{C} 1$ \\
each building & B3 & $\mathrm{C} 1$ & $\mathrm{C} 1$ & $\mathrm{C} 1$ & $\mathrm{C} 1$ \\
\hline
\end{tabular}

All of these vehicles were classified in $\mathrm{C} 1$ for the three buildings, however, the vehicle number four is classed in two different class $\mathrm{C} 1$ and $\mathrm{C} 4$ and this is due to the vehicle-related battery injected power.

\subsection{Database form}

Due to the big database we obtain, we cannot expose all data, therefore, in this part, we present an example of data for four vehicles. Referring to figure (2), those vehicles had numbers from six to three. The corresponding speed, acceleration, and used battery power are as presented in figure (6). We suppose that each vehicle has a unique driving method for three specific situations. $Z_{A}$ correspond to the traffic jam situation and this part is related to the building $\mathrm{A}$. $\mathrm{Z}_{\mathrm{B}}$ corresponds to the bend situation and $Z_{c}$ corresponds to the rest of road which contains a hill climb situation corresponding to the period of time [70s 90s].
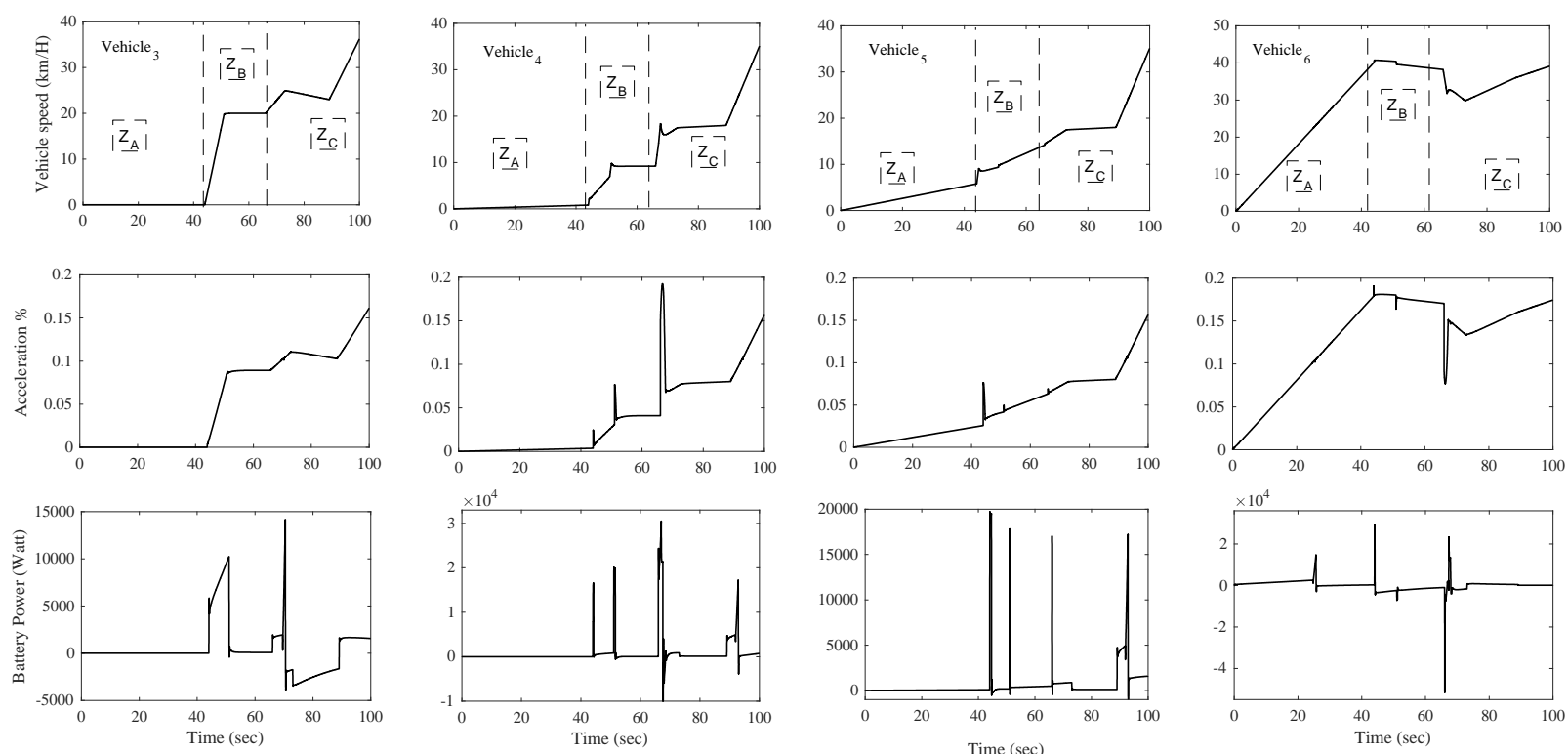

Figure 6. Related speed, acceleration form, and the corresponding battery outputted power for the four cars

As it is mentioned in figure (2), we have supposed that each building (A, B and C), are near to each specific case ( the traffic jam, the left bend and to the hill climb). The given vehicle parameters will be used for the learning phase in the next step.

\subsection{Learning phase}

After classifying four cars, the software application will update its own optimum energetic form according to this database. For this step, the neural network algorithm is used for identifying the optimum acceleration form which corresponds to the optimum energetic consumption. This step is very particular. The optimum energetic consumption can be obtained by an acceleration form close to zero. Our approach does not force the driver to drive with a specified form, but the obtained neural result will limit the acceleration form of the driver in order to obtain the best energetic form. In this situation, we obtain two different cases. 
- In first case: if the given acceleration form is highest then the optimal one. The acceleration form will follow the optimal one.

- In second case: if the given acceleration form is less than the given one. The existing form will be conserved.

The learning phase was obtained by a neural network bloc composed by 4 neurons in the input layers, 3 neurons in the hidden layer and one output neuron in the output layer. The sigmoid function is used as the activation function.

In the given example, three cars were classified in $\mathrm{C} 1$, those data were used for the learning phase and the optimal acceleration form according to this data is exposed in figure (7).

Each building recognizes now the optimal acceleration form that will save energy and those results will be uploaded to the corresponding vehicle if need.
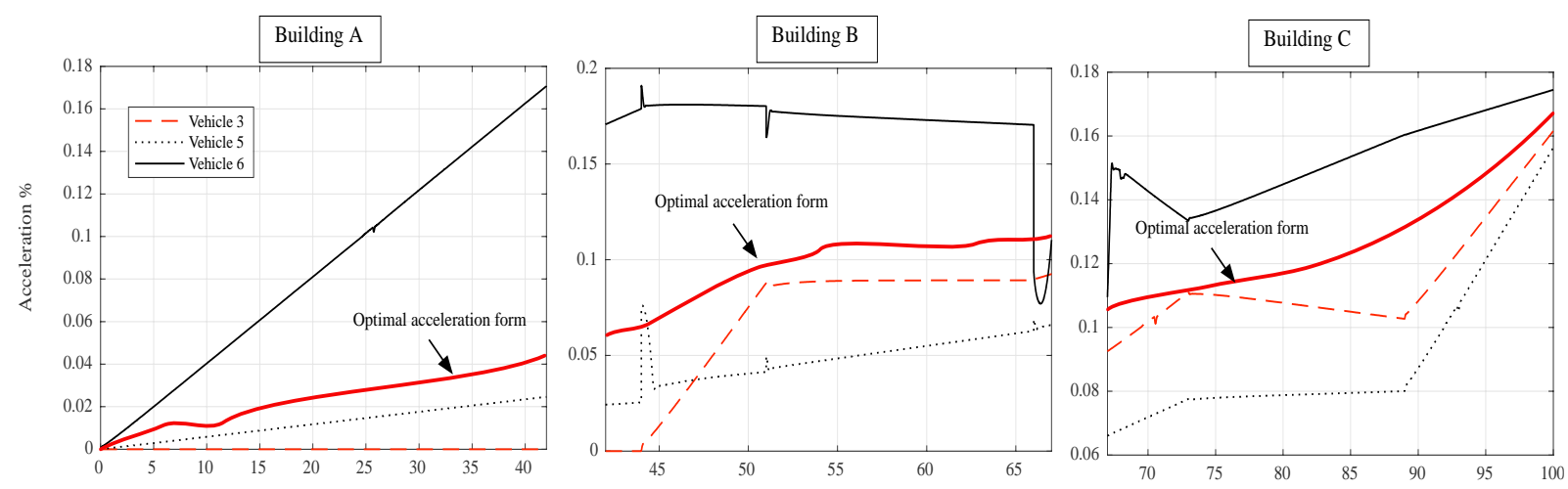

Figure 7. Optimal acceleration form for class $\mathrm{C} 1$

\subsection{Energetic gain}

After having those optimal results, the new vehicle on the road, numbered "vehicle 2" which will pass from building " $\mathrm{A}$ " to building " $\mathrm{C}$ ", will change its acceleration form according to the building recommendation and the new statistics about energy will be as presented in table (6).

The driver was applying an acceleration form as presented in figure (8). If the vehicle is driven in the economic mode, each building will inform the vehicle with optimal solution.

If the vehicle follows this recommendation and does not exceed the optimal solution, then the energetic form will be optimal. For this example, it is clear that the actual acceleration form still under the optimal solution suggested by the algorithm and this is located in the part associated to building " $\mathrm{C}$ " and in another section associated to building " $\mathrm{A}$ ". Consequently, the vehicle acceleration form is conserved in these two regions. However, the reference acceleration form in a special part linked to building " $\mathrm{B}$ " is not recommended and then the new acceleration form (optimal one) will be used. The same application occurs near to building "A". According to this situation, the energy gain will be only in the special parts related to building " $\mathrm{B}$ " and " $\mathrm{A}$ " as it is exposed in figure (8).

The new vehicle experience will be conserved and then the database will be updated and new learning phase will start for updating the new optimal results. 


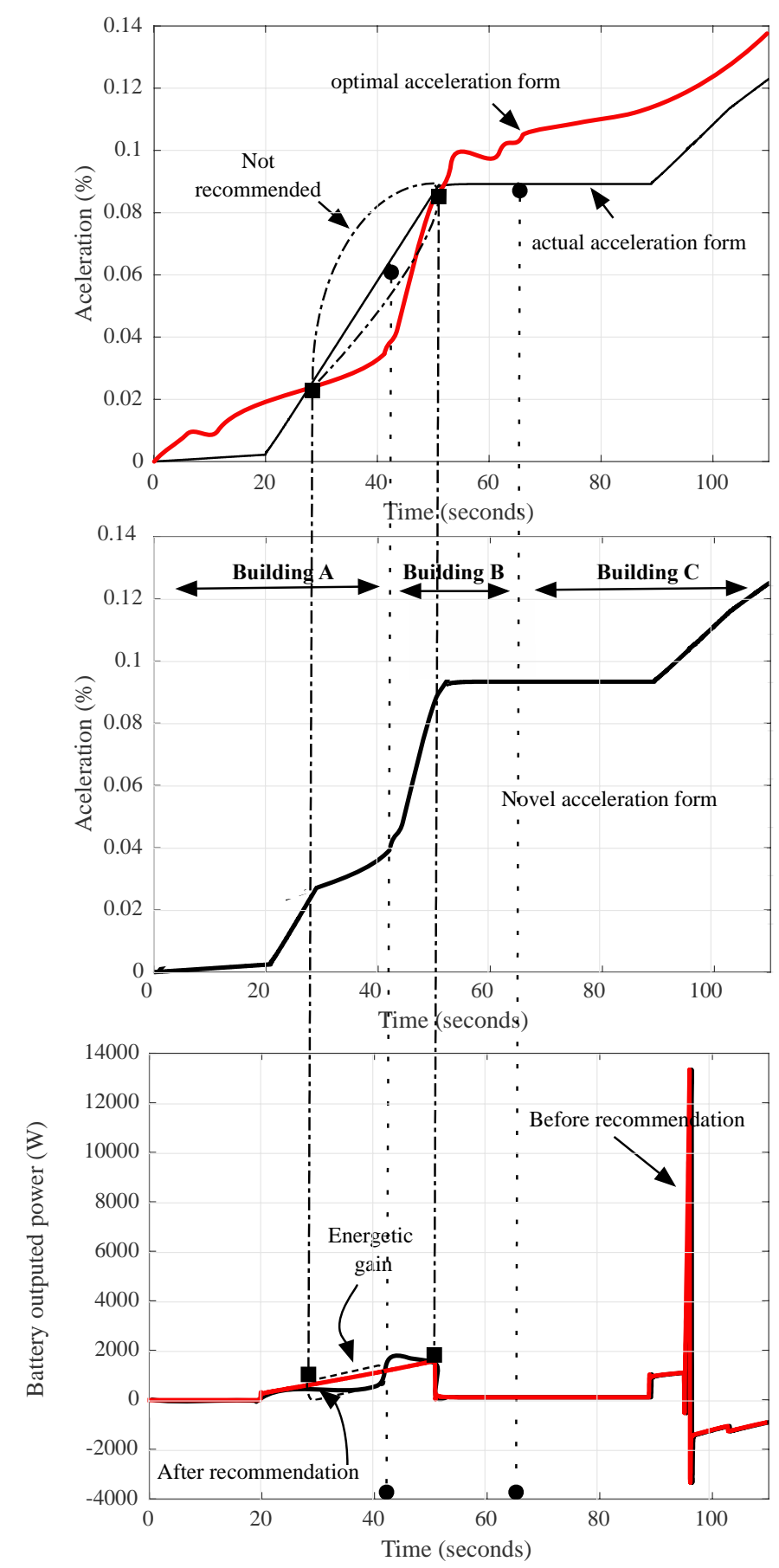

Figure 8. Actual, optimal and novel acceleration form for the vehicle " 2 " and related energetic gain

The same test will be applied on the first vehicle called vehicle " 1 ". However, this vehicle acceleration form will be different from the first case. The driver drives its car with a nervous acceleration form as it is represented in figure (9). In this case, the car will hold two different classes, $\mathrm{C} 1$ and $\mathrm{C} 2$. Two different acceleration forms will be recommended for this vehicle according to the exit data. The vehicle will transit from $\mathrm{C} 1$ to $\mathrm{C} 2$ in the building " $\mathrm{A}$ " region. This transaction form will affect the battery power form as it is presented in the joined results. It is true that, this form is not being recommended. It can be optimized if the classification phase will be extended to more than three categories for each data.

However, if we emphasis on the global energetic form we conclude that the new form is more economic. 


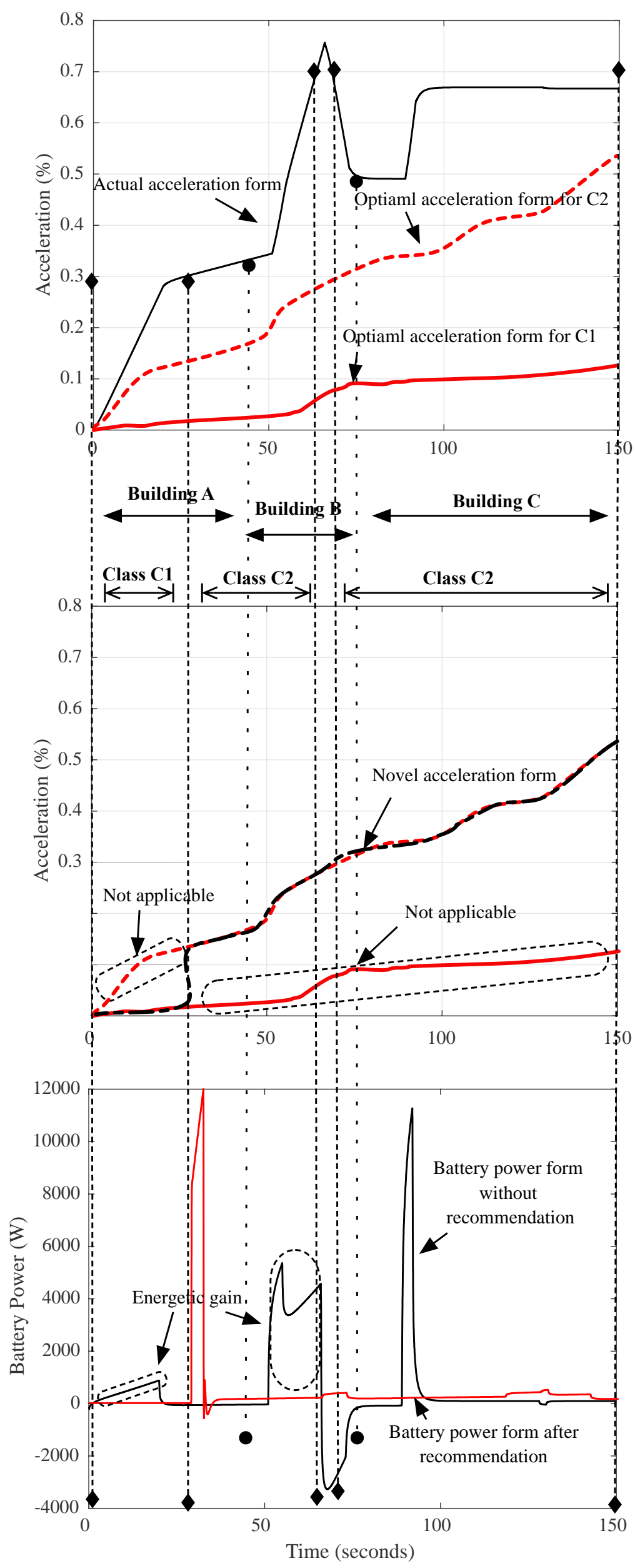


Figure 9. Actual, optimal and novel acceleration form for the vehicle " 1 " and the according to the injected battery power

The provision of this approach can be clearly observed if we focus on the state of charge of the used battery. Therefore, we present in the table (6) the SOC of each car before and after using building recommendations. It is clear that for the two cases, the novel SOC is better than the first situation.

Table 6: SOC before and after the recommendation

\begin{tabular}{cccc} 
& \multirow{2}{*}{ Initial SOC } & Before recommendation & After recommendation \\
\cline { 2 - 4 } Vehicle 2 & $100 \%$ & $99,1 \%$ & $99,3 \%$ \\
Vehicle 1 & $100 \%$ & $97,9 \%$ & $97,5 \%$ \\
\hline
\end{tabular}

\section{Drawbacks of this approach}

Referring to the obtained results, the proposed approach can improve the energy level inside the battery and then the vehicle autonomy. However, this advantage can't hide the drawback of this approach related to several points; To perform such application, we need high power calculators installed in each building and in vehicles. Communication problems can happen between the two systems and redundancy backup system is required for a flawless operation. Finally, a standardization on vehicle's software is needed so more vehicles can be used and more specific energetic date can be collected; more vehicles will define more classes and more efficient and precise Energy optimization.

\section{Conclusions}

Smart cities and efficient transport systems were two specials objectives to develop our daily life. Effectively, increasing electrical vehicle autonomy, force researchers to develop intelligent and robust solutions. Making an intelligent and robust communication system can be seen as a serious objective. In this background, we have developed a novel approach which helps saving energy inside an electrical vehicle. The idea was based on communication between buildings and cars. Indeed, each car will share its own data and each building will transmit to this car the optimal solution which is equivalent to the optimal energetic form. Based on Matlab software we have exposed some results for a specific case and we have shown that with this novel approach an energetic gain can occur.

Author Contributions: Flah. Aymen. did theoretical analysis, system implementation, simulations test and wrote the first original draft paper and write the last version of the draft paper. The proposed approach was discussed with Mahmoudi. Chokri. who verified also the first paper draft. Sbita lassaad was responsible for, supervision and administration, his contribution was related to the theoretical analysis, data analysis, and the paper reviewing and editing.

Funding: "This research received no external funding"

Acknowledgments: This work was selected as the best second paper in ISAECT 2018 conference.

Conflicts of Interest: “The authors declare no conflict of interest." 
Appendix A

\begin{tabular}{|c|c|c|c|c|c|}
\hline $\mathrm{fr}_{\mathrm{r}}$ & The rolling resistance & $\mathrm{Cd}_{\mathrm{d}}$ & Drag coefficient & $\mathrm{m}_{\mathrm{vo}}$ & Vehicle mass \\
\hline $\mathrm{M}_{\mathrm{v}}$ & Mass of the vehicle & $\rho$ & Air density & mba & Battery mass \\
\hline g & Gravitational acceleration & $A_{v}$ & Frontal area of the vehicle & mem & Electric motor mass \\
\hline $\mathrm{Wm}$ & Motor speed & $\mathrm{Q}_{\mathrm{c}}$ & Battery capacity & Ibatt & Battery current \\
\hline$\gamma_{\mathrm{m}}$ & Motor efficiency & $\mathrm{V}_{\mathrm{oc}}$ & Battery voltage & Rbatt & Battery resistance \\
\hline$\theta$ & Road grade & $\mathrm{V}_{\mathrm{v}}$ & Vehicle speed & $\mathrm{C}_{\mathrm{m}}$ & Motor torque \\
\hline
\end{tabular}

\section{References}

1. Author 1, A.B.; Author 2, C.D. Title of the article. Abbreviated Journal Name Year, Volume, page range, DOI.

2. Author 1, A.; Author 2, B. Title of the chapter. In Book Title, 2nd ed.; Editor 1, A., Editor 2, B., Eds.; Publisher: Publisher Location, Country, 2007; Volume 3, pp. 154-196, ISBN.

3. Author 1, A.; Author 2, B. Book Title, 3rd ed.; Publisher: Publisher Location, Country, 2008; pp. 154-196, ISBN.

4. Author 1, A.B.; Author 2, C. Title of Unpublished Work. Abbreviated Journal Name stage of publication (under review; accepted; in press).

5. Author 1, A.B. (University, City, State, Country); Author 2, C. (Institute, City, State, Country). Personal communication, 2012.

6. Author 1, A.B.; Author 2, C.D.; Author 3, E.F. Title of Presentation. In Title of the Collected Work (if available), Proceedings of the Name of the Conference, Location of Conference, Country, Date of Conference; Editor 1, Editor 2, Eds. (if available); Publisher: City, Country, Year (if available); Abstract Number (optional), Pagination (optional).

7. Author 1, A.B. Title of Thesis. Level of Thesis, Degree-Granting University, Location of University, Date of Completion.

8. Title of Site. Available online: URL (accessed on Day Month Year).

(1) Pérez-chacón, R.; Luna-romera, J. M.; Troncoso, A. Big Data Analytics for Discovering Electricity Consumption Patterns in Smart Cities. 2018, 1-19. https://doi.org/10.3390/en11030683. 
(2) Tie, S. F.; Tan, C. W. A Review of Energy Sources and Energy Management System in Electric Vehicles. Renew. Sustain. Energy Rev. 2013, 20, 82-102. https://doi.org/10.1016/j.rser.2012.11.077.

(3) Monteiro, V.; Pinto, J. G.; Afonso, J. L. Operation Modes for the Electric Vehicle in Smart Grids and Smart Homes: Present and Proposed Modes. IEEE Trans. Veh. Technol. 2016, 65 (3), 1007-1020. https://doi.org/10.1109/TVT.2015.2481005.

(4) Rioux, Si.-P. Statistiques on electical vehicle in Canada https://www.aveq.ca/actualiteacutes/category/statistiques.

(5) Karbowski, D.; Sokolov, V.; Jongryeol, J. Fuel Saving Potential of Optimal Route-Based Control for Plug-in Hybrid Electric Vehicle. IFAC-PapersOnLine 2016, 49 (11), 128-133. https://doi.org/http://dx.doi.org/10.1016/j.ifacol.2016.08.020.

(6) Hawkins, T. R.; Gausen, O. M.; Stroemman, A. H. Environmental Impacts of Hybrid and Electric Vehicles-a Review. Int. J. Life Cycle Assess. 2012, 17 (8), 997-1014. https://doi.org/10.1007/s11367-0120440-9.

(7) 10 Longest-Range Electric Vehicles For 2018 https://www.motor1.com/features/228379/longest-rangeevs/2850064/ (accessed Oct 16, 2018).

(8) Khan Ali L Badjate Assistant Professor, Z. S.; Kshirsagar, R. V. Review on Energy Management System for Hybrid Vehicle. IJSTE -International J. Sci. Technol. Eng. 2016, 2 (12), 95-98.

(9) Zhang, S.; Xiong, R. Adaptive Energy Management of a Plug-in Hybrid Electric Vehicle Based on Driving Pattern Recognition and Dynamic Programming. Appl. Energy 2015, 155, 68-78. https://doi.org/10.1016/j.apenergy.2015.06.003.

(10) Mahmoudi, C.; Flah, A.; Sbita, L. Prototype Design of a Compact Plug-in Solar Electric Vehicle Application for Smart Power Management Architecture. In International Conference on Green Energy and Conversion Systems (GECS); 2017. https://doi.org/10.1109/GECS.2017.8066162.

(11) Shetty, S. S.; Karabasoglu, O. Regenerative Braking Control Strategy for Hybrid and Electric Vehicles Using Artificial Neural Networks BT - Engineering Applications of Neural Networks: 15th International Conference, EANN 2014, Sofia, Bulgaria, September 5-7, 2014. Proceedings; Mladenov, V., Jayne, C., Iliadis, L., Eds.; Springer International Publishing: Cham, 2014; pp 103-112. https://doi.org/10.1007/978-3-319-11071-4_10.

(12) Itani, K.; De Bernardinis, A.; Zoubir, K.; Jammal, A. Extreme Conditions Regenerative Braking Modeling, Control and Simulation of a Hybrid Energy Storage System for an Electric Vehicle. IEEE Trans. Transp. Electrif. 2016, 7782 (99), 1-16. https://doi.org/10.1109/TTE.2016.2608763.

(13) Werachet, K.; Heinz, Z. Wirless Power Charging on Electric Vehicles. In international electrical engineering congress; 2014; pp 6-9.

(14) Flah, A. Internal Fuzzy Hybrid Charger System for a Hybrid Electrical Vehicle. ASME, J. Energy Resour. Technol. 2017, 140 (1), 12003-12008. 
(15) Gaoua, Y. On-Line HEV Energy Management Using a Fuzzy Logic. In 12th International Conference on Environment and Electrical Engineering (EEEIC); 2013; p 6.

(16) Panday, A.; Bansal, H. O. A Review of Optimal Energy Management Strategies for Hybrid Electric Vehicle. Int. J. Veh. Technol. 2014, 2014, 19.

(17) Bhati, A.; Hansen, M.; Chan, C. M. Energy Conservation through Smart Homes in a Smart City : A Lesson for Singapore Households. Energy Policy 2017, 104 (January), 230-239. https://doi.org/10.1016/j.enpol.2017.01.032.

(18) Li, B.; Kisacikoglu, M. C.; Liu, C.; Singh, N.; Erol-kantarci, M. Big Data Analytics for Electric Vehicle Integration in Green Smart Cities. 2017, No. November, 19-25.

(19) Helmers, E.; Marx, P. Electric Cars: Technical Characteristics and Environmental Impacts. Environ. Sci. Eur. 2012, 24 (1), 14. https://doi.org/10.1186/2190-4715-24-14.

(20) Azahan, N. A. .; Jamian, J. .; Noorden, Z. . Analysis of Relationship between Acceleration and Battery State of Charging in Electric Vehicle. In IEEE international conference on power and energy; 2016; pp 150154.

(21) Xiong, R.; Cao, J.; Yu, Q.; He, H.; Sun, F. Critical Review on the Battery State of Charge Estimation Methods for Electric Vehicles. IEEE Access 2018, 6, 1832-1843.

https://doi.org/10.1109/ACCESS.2017.2780258.

(22) Young, K.; Wang, C.; Wang, L. Y.; Strunz, K. Electric Vehicle Battery Technologies; Springer International Publishing, 2013. https://doi.org/10.1007/978-1-4614-0134-6.

(23) Calvillo, C. F.; Villar, J. Energy Management and Planning in Smart Cities Energy Management and Planning in Smart Cities. 2016, No. March. https://doi.org/10.1016/j.rser.2015.10.133.

(24) Monitoring, S.; Chui, K. T.; Lytras, M. D. Energy Sustainability in Smart Cities : Artificial. 2018, 1-20. https://doi.org/10.3390/en11112869.

(25) Menouar, H.; Güvenc, I.; Akkaya, K.; Uluagac, A. S.; Kadri, A.; Tuncer, A. UAV-Enabled Intelligent Transportation Systems for the Smart City : Applications and Challenges. 2017, No. March, 22-28.

(26) Letaief, K. B. To Smart City : Public Safety Network Design for Emergency. IEEE access 2018, 6, 14511460.

(27) comment conduire en montée.

(28) conduite dans les virages.

(29) Innerwinkler, P.; Ebner, W.; Stolz, M. Support Vector Machines for Determination of an Operational Strategy for Hybrid Electric Vehicles. 2015 23rd Mediterr. Conf. Control Autom. 2015, No. 6, 709-714. https://doi.org/10.1109/MED.2015.7158829.

(30) Niu, W.; Feng, Z.; Feng, B.; Min, Y.; Cheng, C. Neural Network , Extreme Learning Machine , and 
Support Vector Machine in Deriving Operation Rule of Hydropower Reservoir. 2019. https://doi.org/10.3390/w11010088. 